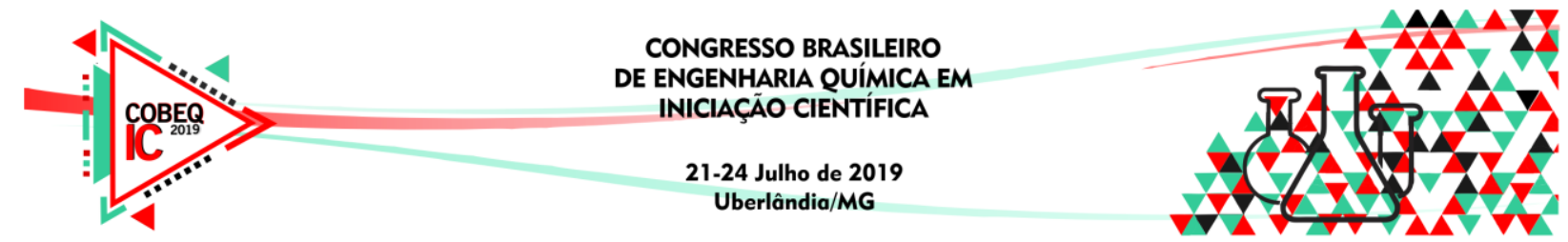

\title{
IDENTIFICAÇÃO, CONTROLE E SINTONIA DE TANQUES EM SÉRIE NÃ̃-INTERATIVOS
}

\author{
J. P. S. FERNANDES ${ }^{1}$, M. O. MARQUES ${ }^{1}$, V. R. DE SOUZA ${ }^{1}$, L. A. DE MEDEIROS ${ }^{1}$, S. \\ F. S. VIDAL ${ }^{1}$ \\ ${ }^{1}$ Instituto Federal do Norte de Minas Gerais - Campus Montes Claros, Faculdade de \\ Engenharia Química \\ E-mail para contato: jpsf@aluno.ifnmg.edu.br
}

\begin{abstract}
RESUMO - Considerando a importância do controle feedback para os processos da indústria química, a proposta desse trabalho foi identificar, analisar a estabilidade e sintonizar sistema de dois tanques de nível em série e nãointerativos, partindo de um set point inicial de $\mathrm{h}=14,62 \mathrm{~cm}$. Na identificação, a bomba 1 que controla a vazão do primeiro tanque apresentou maior constante de ganho que a da bomba 2 que regula a vazão de líquido do mesmo tanque, assim, verifica-se que a resposta da primeira bomba foi mais rápida. Pelo método CohenCoon encontrou-se constante do controlador de 69,49 e, na sintonia fina, variando-se para mais $(\mathrm{Kc}=83,56)$ percebeu-se aumento de oscilação e resposta mais rápida sendo, portanto, o valor mais adequado para o processo.
\end{abstract}

\section{INTRODUÇÃO}

A engenharia de controle integra o uso de estratégias de projetos para aprimorar processos industriais, considerando a compreensão e modelagem dos sistemas, objetivando aumentar a eficiência dos mesmos e a diminuir custo (DORF et al., 1998). Segundo Stephanopoulos (1984) durante uma operação, uma planta química deve satisfazer algumas exigências como, por exemplo, econômicas, sociais, ambientais, entre outros, por isso sistemas de controle são inseridos de modo que possíveis distúrbios no processo possam ser eliminados ou neutralizados e para que se tenha um monitoramento constante das principais variáveis.

As variáveis envolvidas na modelagem, estratégia utilizada para possibilitar a simulação do sistema, são denominadas variáveis de controle e variáveis manipuladas. Um sistema de controle comumente usado é o Feedback que é definido por Coughanwor (1991) como um controle que envolve o uso da variável controlada para manter o processo em um valor desejado ou Set Point. Diante disso, nesse trabalho foi feito o estudo de um sistema de tanques em série, não-interativos em malha aberta e fechada para, assim, determinar a ação de controle adequada juntamente com análise de estabilidade e sintonia fina do processo.

\section{METODOLOGIA}

Foi utilizado um módulo contendo dois tanques em série não-interativos nos quais existem duas bombas que impulsionam o líquido (água) para o primeiro tanque (T-01) e deste o fluido era bombeado para o segundo tanque (T-02) via mangueira, conforme a Figura 1. 


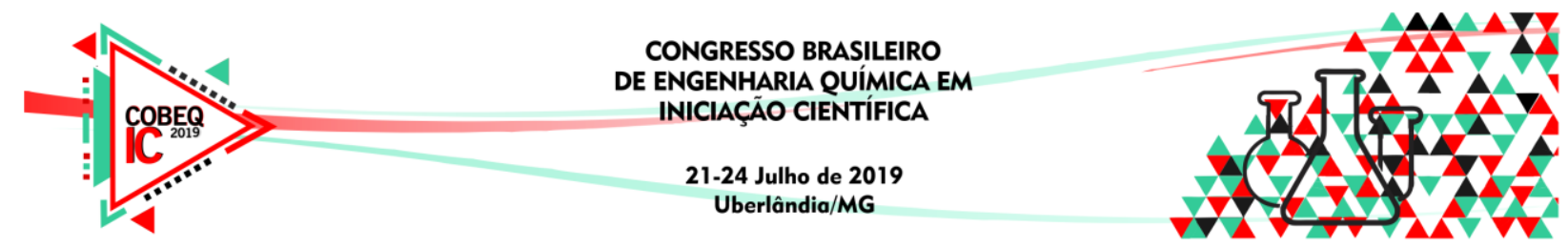

Figura 1 - Módulo com dois tanques de níveis e duas bombas

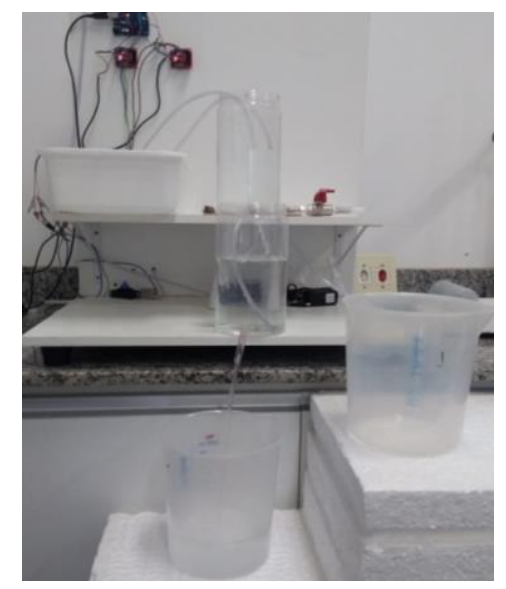

O processo de calibração foi feito a partir de ajuste linear entre as alturas emitidas pelo sensor e a altura real medida através de uma régua milimetrada. Assim, os parâmetros obtidos a partir da reta foram usados em todos os resultados do trabalho.

O sistema representado na Figura 1 teve uso possibilitado pela junção do módulo XCos da plataforma livre Scilab com transmissão e autuação promovida por placa de Arduino UNO conectada às bombas utilizadas no processo. Através de sinais transmitidos às bombas, elas autuavam conforme as perturbações/distúrbios provocados para estudo do sistema.

Em controle de processos, o estudo da dinâmica é realizado a partir de variáveis-desvio, sendo assim, é essencial avaliar o estado estacionário. Dessa forma, configurou-se as bombas conforme a Tabela 1 e para a respectiva configuração analisou-se o estado estacionário no gráfico obtido no Scilab mostrado na Figura $2 b$.

Tabela 1 - Configuração para obtenção do estado estacionário

\begin{tabular}{cccc}
\hline Parâmetro & $\mathbf{B}_{\mathbf{1}}(-)$ & $\mathbf{B}_{\mathbf{2}}(-)$ & $\mathbf{t}(\mathbf{s})$ \\
\hline Valor & 102 & 127,5 & 600 \\
\hline
\end{tabular}

Após determinar o estado estacionário, realizou-se a análise em malha aberta. Sendo assim, frente à perturbação (Tabela 2) e ao distúrbio (Tabela 3) com modificação das configurações de $\mathrm{B}_{1}$ e $\mathrm{B}_{2}$, obteve-se o gráfico e os parâmetros de ganho $\left(\mathbf{K}_{\mathbf{i}}\right)$, constante de tempo $(\boldsymbol{x})$ e tempo morto do processo $(\mathbf{t d})$.

Tabela 2 - Configuração para obtenção da função de transferência da perturbação

\begin{tabular}{cccc}
\hline Parâmetro & $\mathbf{B}_{1}(-)$ & $\mathbf{B}_{2}(-)$ & $\mathbf{t}(\mathbf{s})$ \\
\hline Valor & 76,5 & 127,5 & 673 \\
\hline
\end{tabular}

Tabela 3 - Configuração para obtenção da função de transferência do distúrbio

\begin{tabular}{cccc}
\hline Parâmetro & $\mathbf{B}_{\mathbf{1}}(-)$ & $\mathbf{B}_{\mathbf{2}}(-)$ & $\mathbf{t}(\mathbf{s})$ \\
\hline Valor & 102 & 102 & 514 \\
\hline
\end{tabular}

Realizou-se a iteração com o solver do Excel utilizando como função-objetivo a variância entre o desvio da altura calculada ( $h$ 'calc) e o desvio da altura (h') para obtenção dos parâmetros, conforme mostra a equação 1. Os gráficos gerados foram adaptados para 


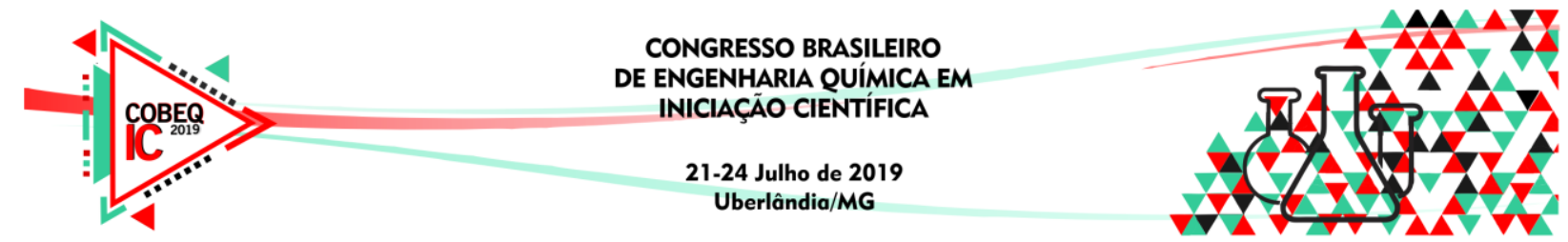

variáveis-desvio e o processo de segunda ordem (tanques em série) foi aproximado a processo de primeira ordem com tempo morto. Posteriormente, empregou-se a relação de Padé para linearizar o termo exponencial no domínio de Laplace, assim como descrito na equação 2 (de modo a facilitar a análise da estabilidade). $\mathrm{O}$ índice $\mathbf{i}$ representa a função de transferência do $\left(\mathrm{G}_{\mathrm{p}}\right.$ e $\left.\mathrm{K}_{\mathrm{p}}\right)$ ou do distúrbio $\left(\mathrm{G}_{\mathrm{d}}\right.$ e $\left.\mathrm{K}_{\mathrm{d}}\right)$.

$$
\begin{aligned}
& h_{\text {cale }}^{\prime}=\left\{\begin{array}{c}
0, \text { se } \tau<t d \\
\left(A K_{i}\left(1-e^{\frac{-(t-t d)}{\tau}}\right), \text { se } \tau>t d\right.
\end{array}\right. \\
& G_{i}=\frac{K_{i}}{\tau s+1} e^{-t d s}=\frac{K_{i}}{\tau s+1}\left(\frac{1-\frac{t d s}{2}}{1+\frac{t d s}{2}}\right)
\end{aligned}
$$

Depois de obtidas $\mathbf{G p}$ e $\mathbf{G d}$, foi possível verificar o comportamento do processo para controle feedback proporcional. Para o caso relatado nesse trabalho, assume-se o valor unitário para as funções de transferência do sensor-transmissor $(\mathbf{G m})$ e atuador $(\mathbf{G v})$, com isso a resposta em malha fechada reduz-se à equação 3.

$$
\mathrm{h}^{\prime}=\frac{G c G p}{1+G c G p} h_{s p}^{\prime}+\frac{G d}{1+G c G p} d^{\prime}, G c=K c
$$

A análise da estabilidade foi realizada pelo método da substituição direta (i $\omega$ ) no polinômio presente no denominador da equação 3 da variável de resposta.

Tabela 4 - Configuração de controle pelo Cohen-Coon $(0<t d / \tau<1)$

\begin{tabular}{cccc}
\hline Tipo de controle & Kc & $\tau_{\mathbf{I}}$ & $\tau_{\mathbf{D}}$ \\
\hline $\mathrm{P}$ & $\frac{\tau}{t d K}\left(1+\frac{t d}{3 \tau}\right)$ & $\infty$ & -- \\
$\mathrm{PI}$ & $\frac{\tau}{t d K}\left(0,9+\frac{t d}{12 \tau}\right)$ & $\frac{t d(30+3 t d / \tau)}{9+20 t d / \tau}$ & -- \\
$\mathrm{PID}$ & $\frac{\tau}{t d K}\left(\frac{16 \tau+3 t d}{12 \tau}\right)$ & $\frac{t d(32+6 t d / \tau)}{13+8 t d / \tau}$ & $\frac{4 t d}{11+2 t d / \tau}$ \\
\hline
\end{tabular}

Além da análise de estabilidade realizou-se a sintonia do processo, onde empregou-se o método de Cohen-Coon e, a partir dos parâmetros de ganhos e constantes de tempo descobertos, utilizou-se a Tabela 4 e descobriu-se um novo Kc.

Após encontrado o novo valor de Kc, realizou-se a sintonia fina, que consiste no ajuste de parâmetros através de ensaios experimentais com valores próximos (20\% maior e 50\% menor) do calculado na sintonia. Calcula-se os erros associados e, a partir dos mesmos, verifica-se qual teve menor somatório e, assim, melhor Kc para o sistema. Considerando as limitações instrumentais (bombas que podem danificar com modificações bruscas) e o tipo de processo (tanques de níveis para armazenamento não necessitam serem rápidos/subamortecidos), o critério utilizado foi o ITAE (Integral of Time multiplied by Absolute of the Error), que penaliza os erros que persistem ao longo do tempo (oscilações). A equação 


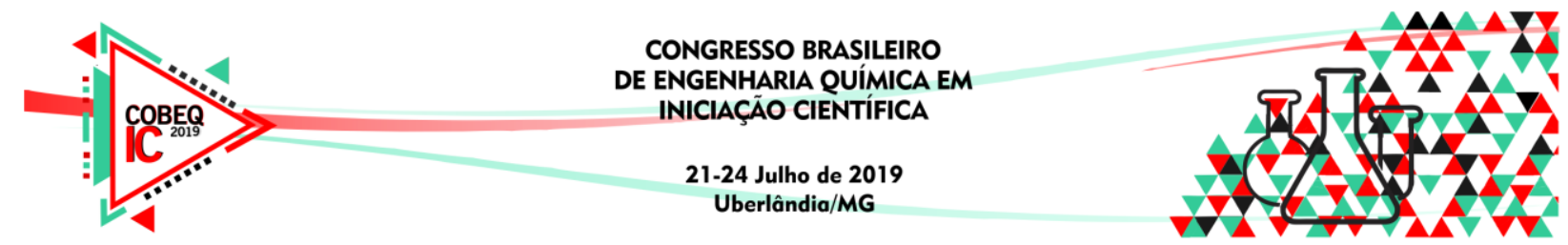

utilizada para análise foi a 4 e o método computacional de integração numérica foi o de trapézios (equação 5).

$$
\begin{gathered}
\int_{0}^{t} t|e| d t \\
\operatorname{ITAE}=\frac{\Delta t}{2}\left(f(1)+2 \sum_{i=2}^{n} f(i)+f(n+1)\right)
\end{gathered}
$$

\section{RESULTADOS E DISCUSSÃO}

A Figura 2a mostra que, realmente, existe divergência dos valores reais com os lidos pelo sensor. Sendo assim, a partir de coeficiente de correlação próximo a 1 mostra que a expressão obtida pelo ajuste linear pode ser aplicada para correção dos dados lidos. O estado estacionário, conforme configuração na Tabela 1 , foi obtido e teve valor de 14,62 cm, como pode ser conferido no gráfico apresentado na Figura 2b. Nota-se que 400 segundos foram suficientes para encontrar o set point inicial.

Figura 2 - (a) Calibração da $1^{\text {a }}$ parte Experimental; e, (b) Estado Estacionário

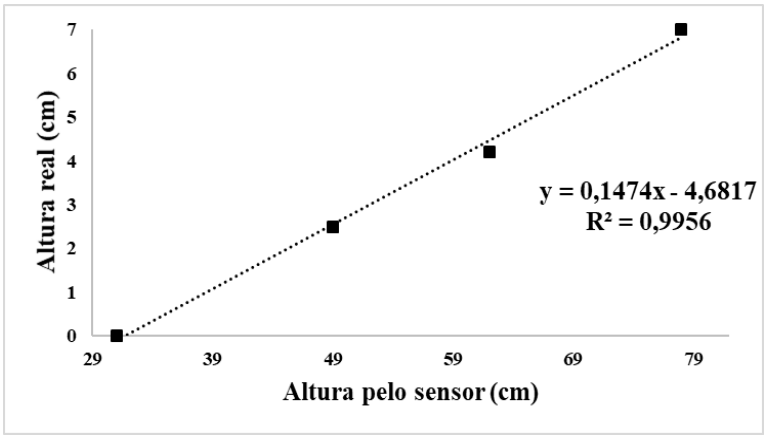

(a)

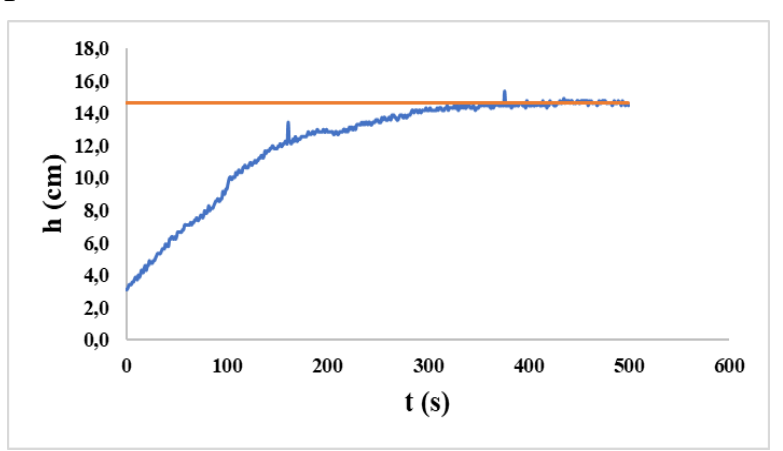

(b)

A identificação do processo foi realizada para duas situações. No primeiro caso, utilizou-se os parâmetros com os valores mostrados na Tabela 2, essa perturbação foi considerada como uma mudança qualquer no set point e, portanto, a função de transferência obtida pôde ser configurada como sendo a do processo, $\mathrm{Gp}(\mathrm{Kp}, \tau, \mathrm{td})$. A partir do gráfico e do solver do Excel $^{\circledR}$, encontrou-se os parâmetros dispostos na Tabela 5.

Nota-se que o ganho do processo é, em relação a outro obtido na análise, baixo. $\mathrm{O}$ fato da constante de tempo ser maior que o tempo morto reafirma a possibilidade de se utilizar o método Cohen-Coon na sintonia, quando se fecha a malha. O processo apresenta resposta rápida e frente à perturbação o desvio tendeu a -2,33. Com isso o formato de Gp fica disposto assim como mostrado na equação 6.

$$
G p=\frac{0,0913}{96,48 s+1} e^{-16,52 s}
$$

Tabela 5 - Parâmetros do processo para perturbação na bomba 1

\begin{tabular}{ccccc}
\hline Parâmetro & $\mathrm{A}$ & $\mathrm{K}_{\mathrm{p}}$ & $\boldsymbol{\tau}$ & td \\
\hline Valor & $-25,5$ & 0,0913 & 96,48 & 16,52 \\
\hline
\end{tabular}



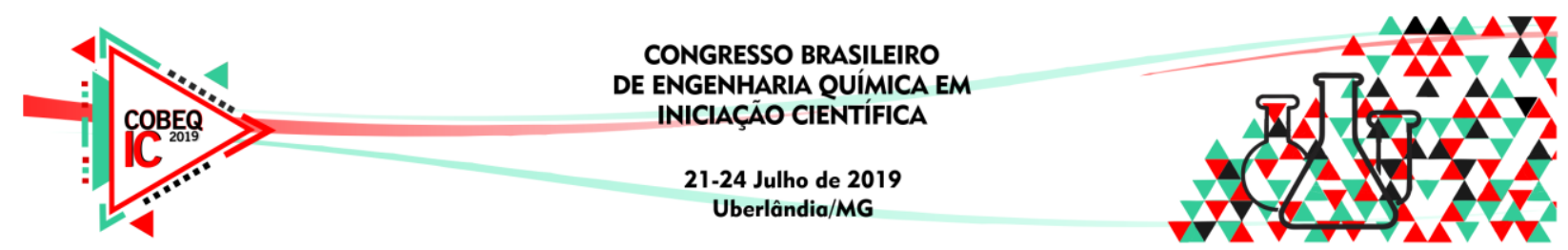

O gráfico mostrado na Figura $3 b$ indica que, em variável-desvio, a alteração na configuração da Tabela 3 ( $\mathrm{B}_{1}$ igual a do estado estacionário; $\mathrm{B}_{2}$ com 25,5 unidades para menos) teve maior alteração no set point inicial do que quando usados os dados da Tabela 2 ( $\mathrm{B}_{1}$ com 25,5 unidades para menos; $\mathrm{B}_{2}$ igual a do estado estacionário). É possível ser verificado também que o ganho para o segundo caso foi maior e a constante de tempo também foi menor, mostrando que o processo foi mais rápido que o anterior.

Figura 3 - Comportamento frente a: (a) Perturbação; e, (b) Distúrbio

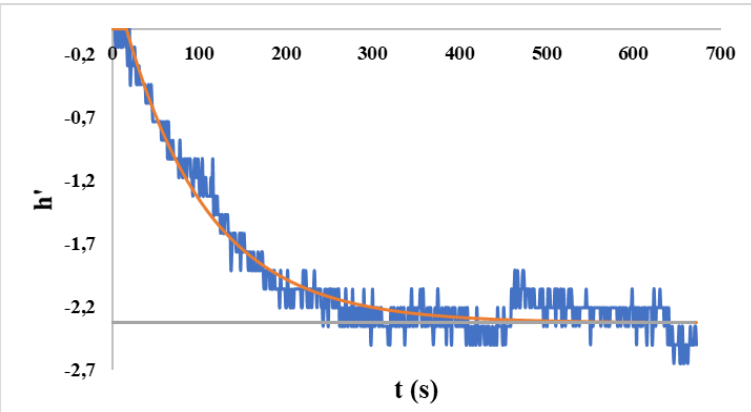

(a)

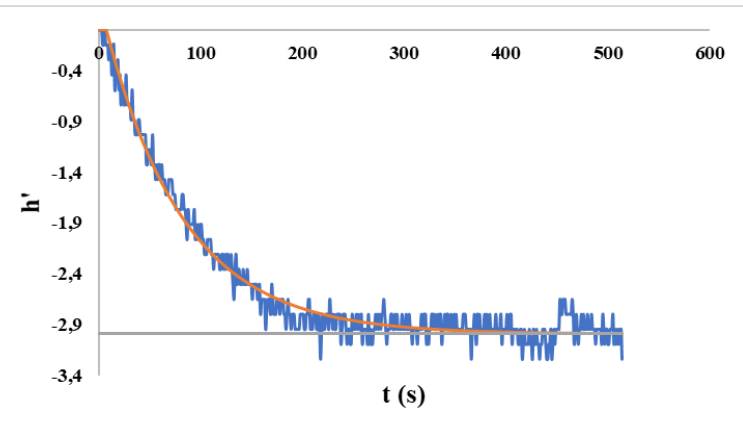

(b)

Através da soma erros ao quadrado pôde-se utilizar o solver do Excel para encontrar os parâmetros da função de transferência do distúrbio (Tabela 6) e, assim, montar a equação 7.

Tabela 6 - Parâmetros do processo para distúrbio na bomba 2

\begin{tabular}{ccccc}
\hline Parâmetro & $\mathrm{A}$ & $\mathrm{K}_{\mathrm{d}}$ & $\tau$ & td \\
\hline Valor & -20 & 0,1497 & 77,71 & 7,70 \\
\hline
\end{tabular}

Pela comparação numérica entre os parâmetros da perturbação e do distúrbio fica evidente que o ganho foi maior e a constante de tempo foi menor. No processo com tanques de nível não é comum a troca de set point, dessa forma, as variações nesse sistema ocorrerão, de forma mais recorrente, como resposta a possíveis distúrbios.

$$
G d=\frac{0,149}{77,71 s+1} e^{-7,7 s}
$$

Figura 4 - (a) Calibração da $2^{\mathrm{a}}$ parte experimental; e, (b) Sintonia Fina

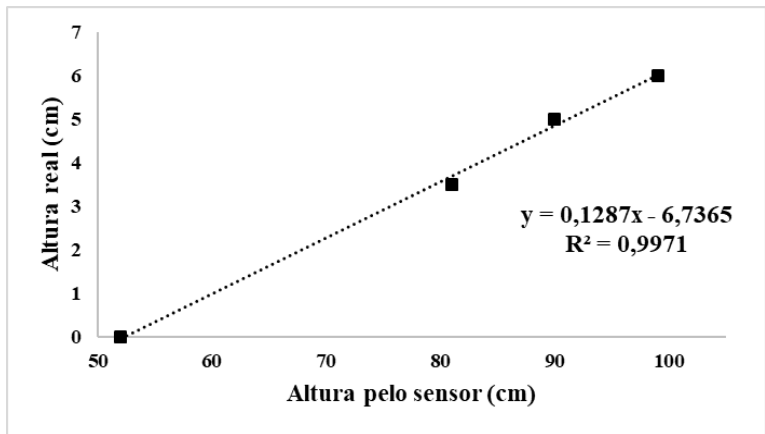

(a)

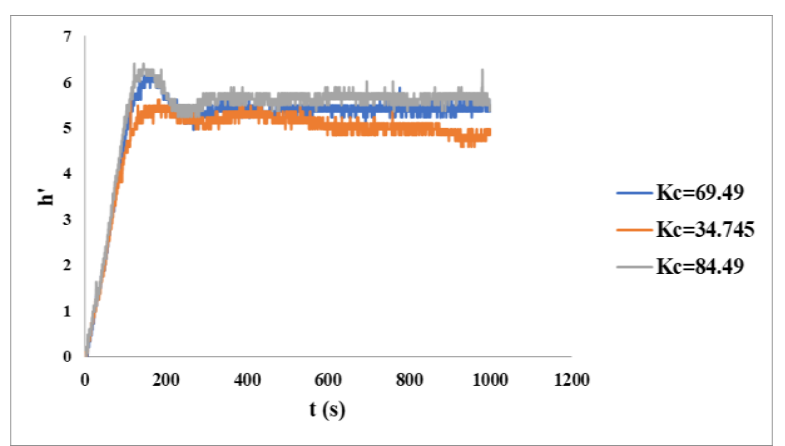

(b)

Assim como na Parte Experimental I, realizou-se a calibração dos instrumentos. Isso foi necessário devido ao fato dos ensaios terem sido realizados em dias distintos. A equação e a 


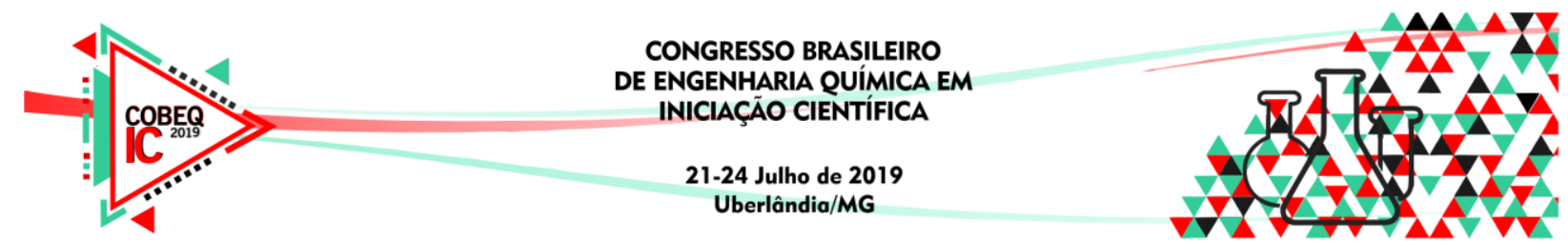

figura do ajuste linear realizado constam na Figura 4a. Pela Figura 4b é possível realizar a comparação do comportamento dos processos para as diferentes constantes, repara-se que maiores Kc implicou no aumento da oscilação, porém, não foram discrepâncias significativas a ponto de inviabilizar o uso do maior valor. O valor de Kc pela Tabela 4 foi 69,49 , nota-se que aumentando-se em $20 \%$ da constante sugerida pelo Cohen-Coon, houve maior rapidez na resposta apontando 83,56 como um ótimo valor na sintonia fina do processo. A diminuição em 50\% apresentou comportamento semelhante, porém, mesmo com valor de Kc menor ao calculado o processo apresentou oscilações deixando clara a inviabilidade de uso.

\section{CONCLUSÃO}

Os estudos realizados neste trabalho objetivaram determinar quantitativamente as variáveis envolvidas na ação de controle adequada para um determinado processo em série, assim como determinar faixa de estabilidade e a melhor sintonia para o processo afim de melhoras os parâmetros para obtenção de melhores resultados.

Assim, considerando o processo foi determinado o uso do controle $\mathrm{P}$ aplicando a ele primeiramente a sintonia do tipo Cohen-Coon e utilizando como sintonia fina o método de integração do erro ao longo do tempo (ITAE) para determinar os valores dos ganhos do processo e do controle bem como a constante de tempo envolvida e demais variáveis pertinentes para o processo. Para tais cálculos foi considerado uma aproximação de $1^{\mathrm{a}}$ ordem para um processo originalmente de $2^{\mathrm{a}}$ ordem.

\section{REFERÊNCIAS}

COUGHANOWR, D. R. et al. Process systems analysis and control. New York: McGrawHill, 1991.

DONALD R. C. Process Systems Analysis and Control. Departament of Chemical ngineering Drexel university. 2 ed. Copyright 1991.

DORF, R. C.; BISHOP, R. H. Sistemas de Controle Modernos. Traduzido por Bernardo Severo da Silva Filho. LTC Editora, 2001. Disponível em: 〈https://www.academia.edu/ 34588989/Sistemas_de_Controle_Modernos_8a_Ed_Dorf_Editora_LTC>. Acesso em: Janeiro, 2019

DUTRA. J. VII. Projeto de Controladores Feedback. ENG -10933 Controle de Processos, 2017

STEPHANOPOUlOS, G. Chemical Process Control na Introduction to Theory and Pratice. Prentice Hall, 1984. 